\title{
Evaluation of Straylight Characteristics of the Sentinel-5 NIR Spectrometer Optics
}

P. Petruck, M. Merschdorf, L. Platos, B. Michel, B. Harand ICSO 2018, 11 th of October 2018
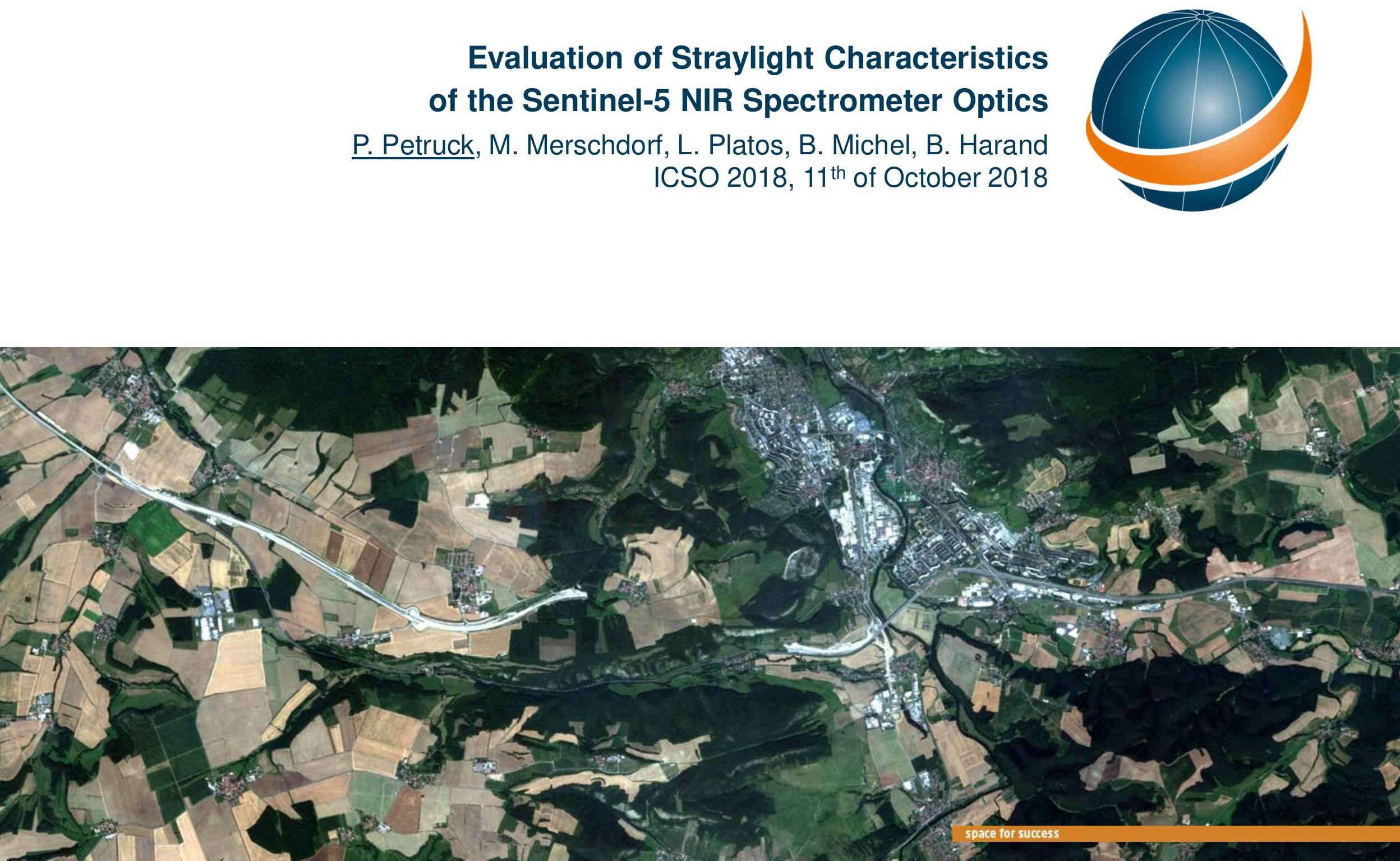


\section{Sentinel-5 mission}

- Sentinel-5 is an atmospheric monitoring mission within the European Copernicus program

- main objective of the mission is the operational global monitoring of trace gas concentrations for atmospheric chemistry and climate applications

- accurate measurements of key atmospheric constituents such as ozone, nitrogen dioxide, sulphur dioxide, carbon monoxide, methane, formaldehyde, and aerosol properties

- Satellite: MetOp-SG A (incl. 8 instruments in total)

- Orbit type: LEO, polar, sun-synchronous (817 km)

- program by European Space Agency (ESA)

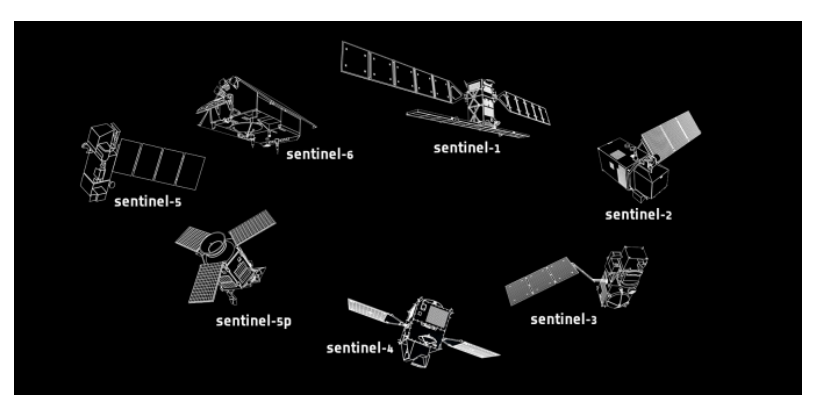

- instrument prime: Airbus Defence \& Space

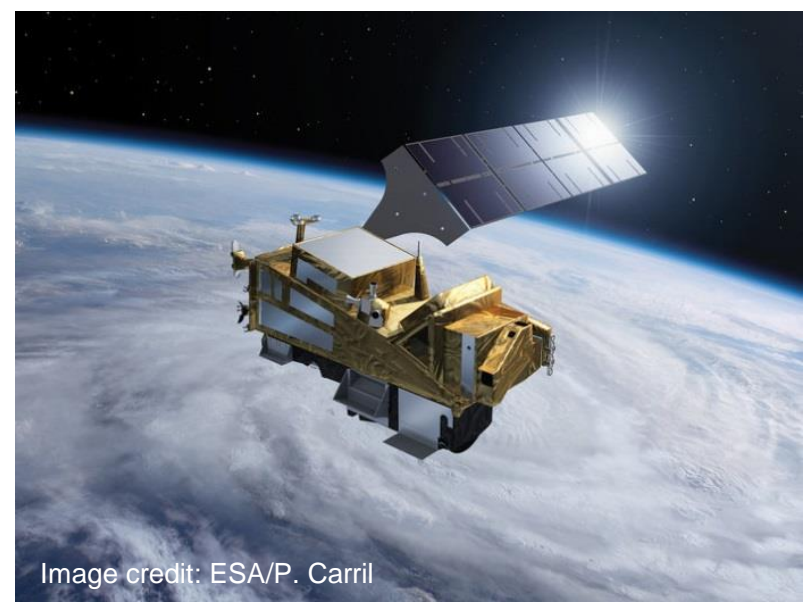




\section{Sentinel-5 instrument}

- Main characteristics

- Type: passive grating imaging spectrometer with two co-aligned telescopes

- Configuration: push broom staring (non-scanning) in nadir viewing

- Spectral: 5 spectrometers (UV1, UV2VIS, NIR, 2x SWIR)

- Swath width: $2670 \mathrm{~km}$; Spatial sampling: $7.5 \times 7.5 \mathrm{~km}^{2}$ (NIR)

- Radiometric: resolution SNR >200 (NIR-1) >520 (NIR-2); 3\% abs. accuracy

- Design lifetime: $>7.5$ years

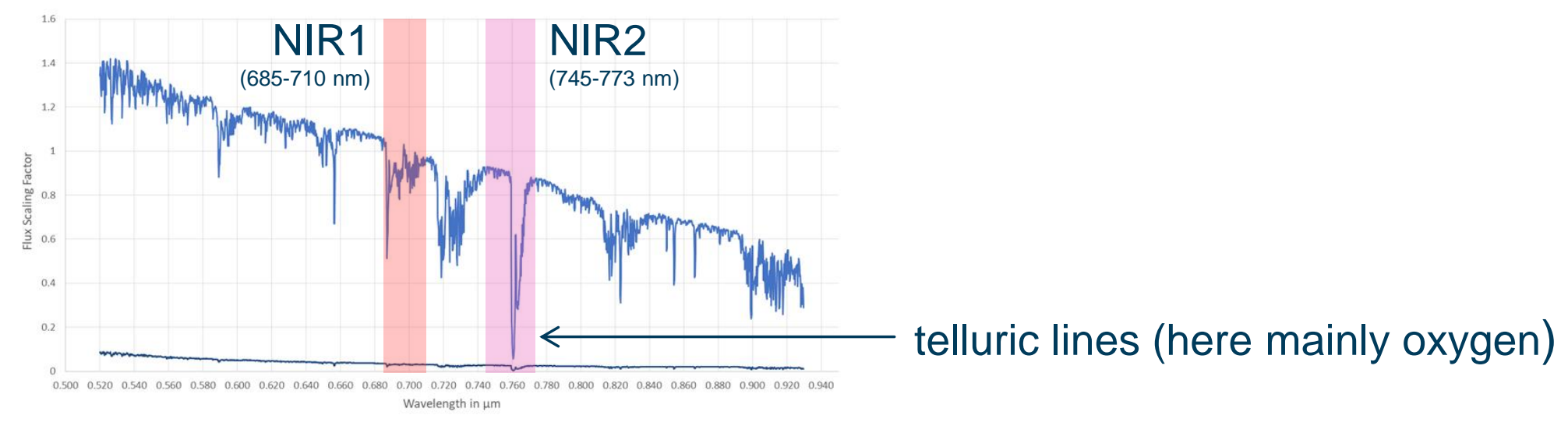




\section{Sentinel-5 NIR spectrometer optics (CDR status)}

- The NIR spectrometer optics is a subsequent assembly after a telescope, a slit and a dichroic beam splitter separating UV-VIS and NIR band wavelength spectrum

- It is purely based on refractive optics (except folding mirrors) and makes use of a grating as dispersive element and a prism for aberration correction

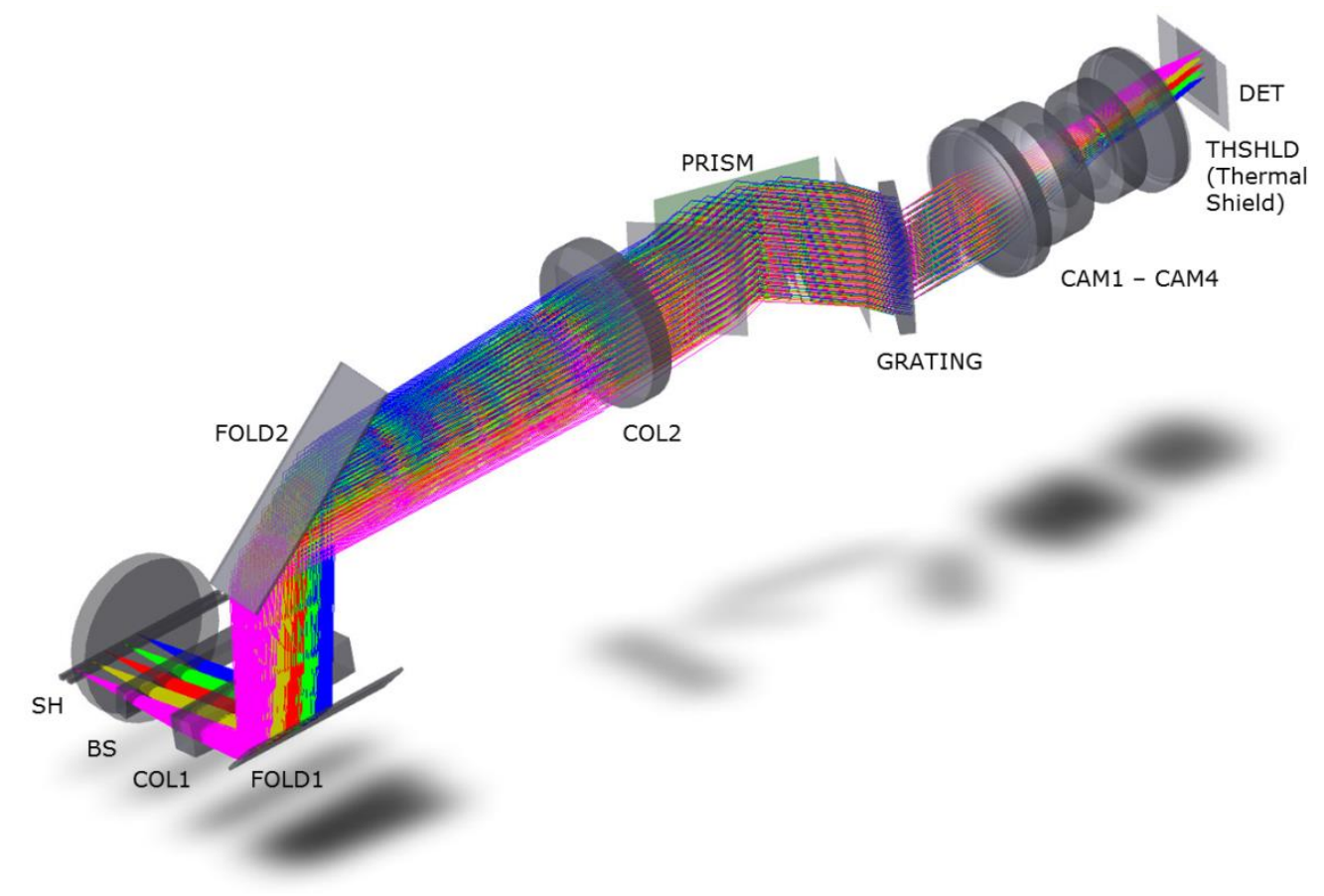




\section{Sentinel-5 NIR spectrometer opto-mechanics (CDR status)}

- The opto-mechanical design is consequently driven by high-accurracy mounting requirements as well as the goal to minimize straylight

- The design comprises

- use of stops and vanes in adequate geometries, where necessary and useful

- high performance anti-reflection coatings on all optical elements

- high performance black coatings on all mechanical elements

- lowest levels of surface roughness for optical surfaces

- lowest levels of particle contamination

- General constrains

- input spectrum (which is broader than the bands of interests)

- beam splitter spectral properties

- reflectivity of detector and thermal shield

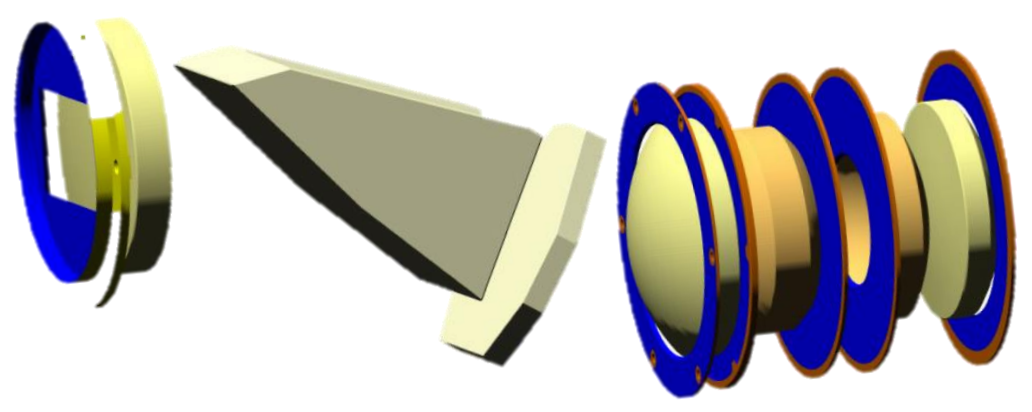




\section{ASAP Model Setup}

- Input models (Zemax and CAD) imported to ASAP and merged

- Designation of particular surfaces as objects, (refractive) media, surface coatings, and BSDF

- Setup grating model including efficiencies, scattering properties for both, transmission and reflection

$\Rightarrow 0^{\text {th }}$ and $-1^{\text {st }}$ order in transmission

$\Rightarrow-2^{\text {nd }},-1^{\text {st }}, 0^{\text {th }},+1^{\text {st }}$ in reflection

- Setup source due to predefined input scene (bright half, dark half of spectrometer slit)

- Raytraces with up to $10^{5}$ rays per wavelength
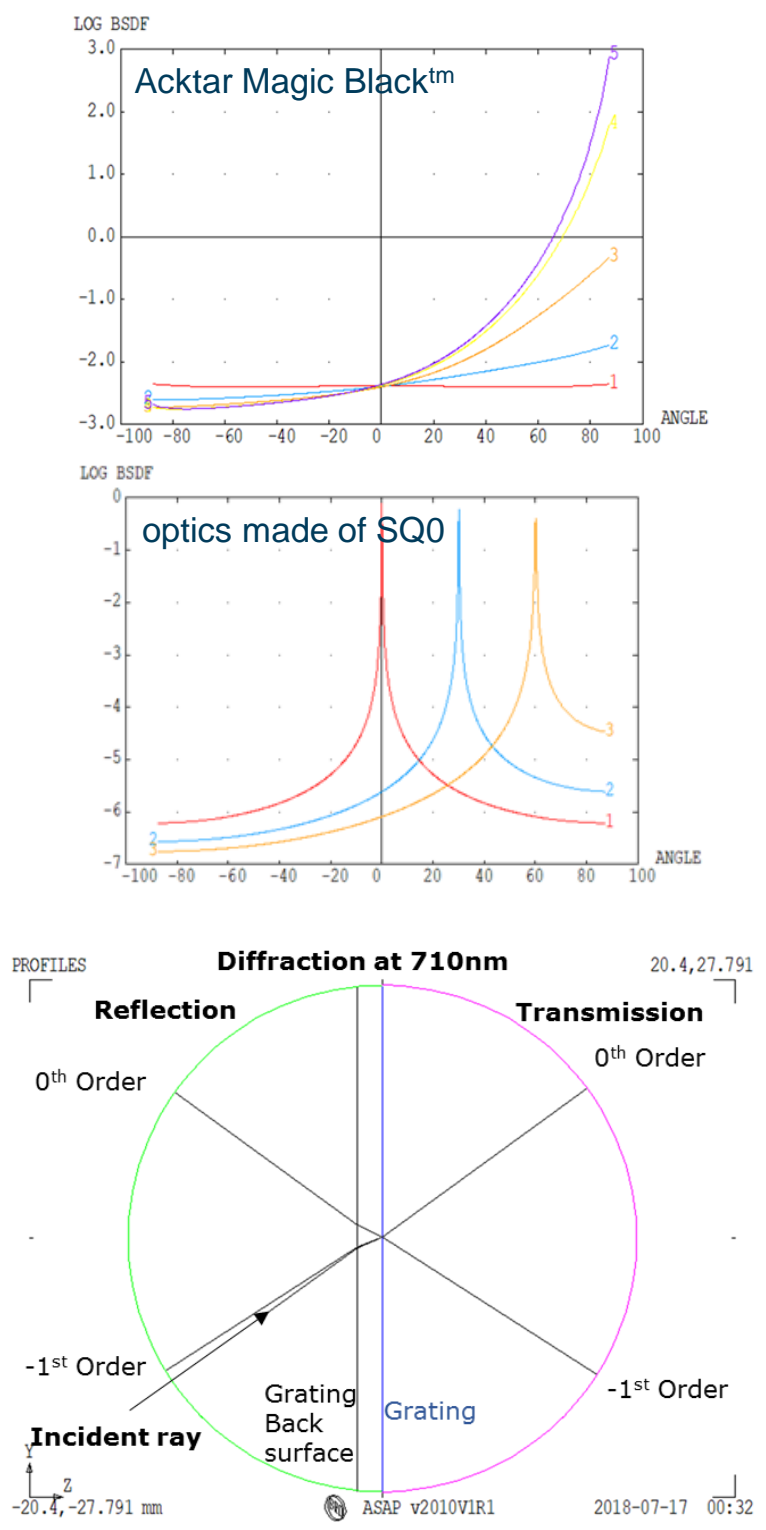


\section{Nominal and direct light}

test scene with full input spectrum

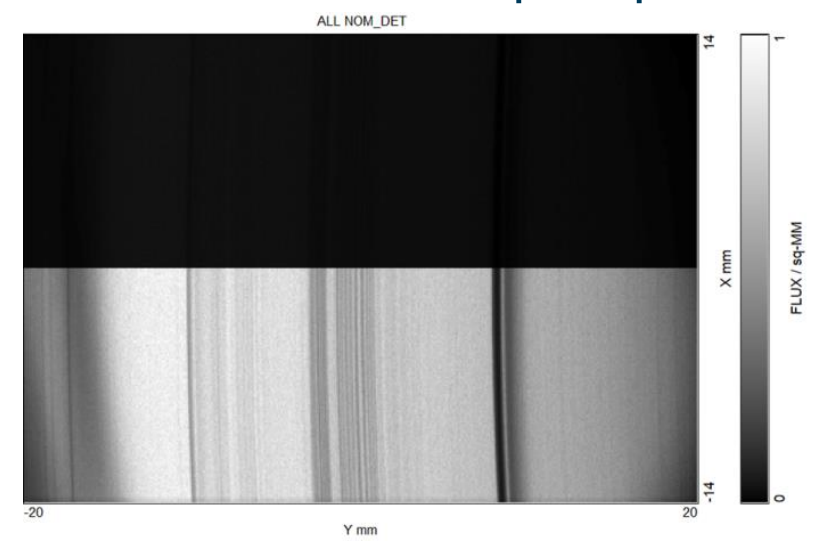

... factor 20x in brightness

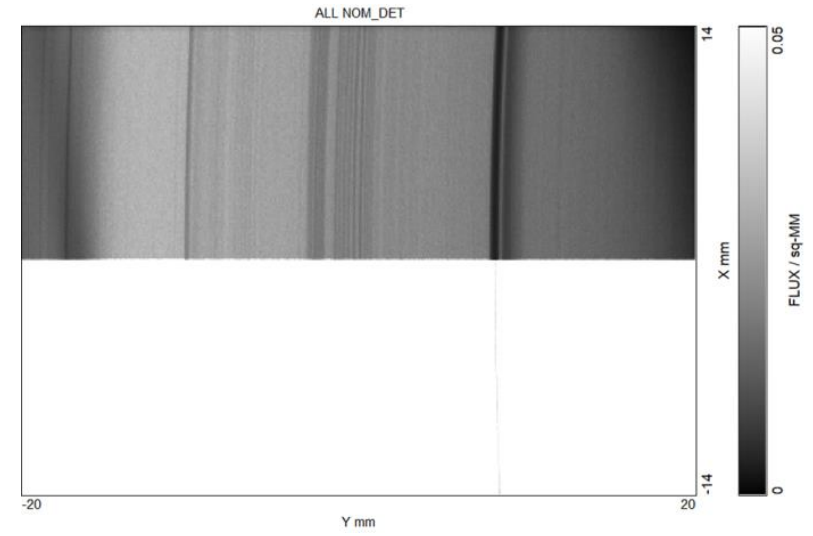

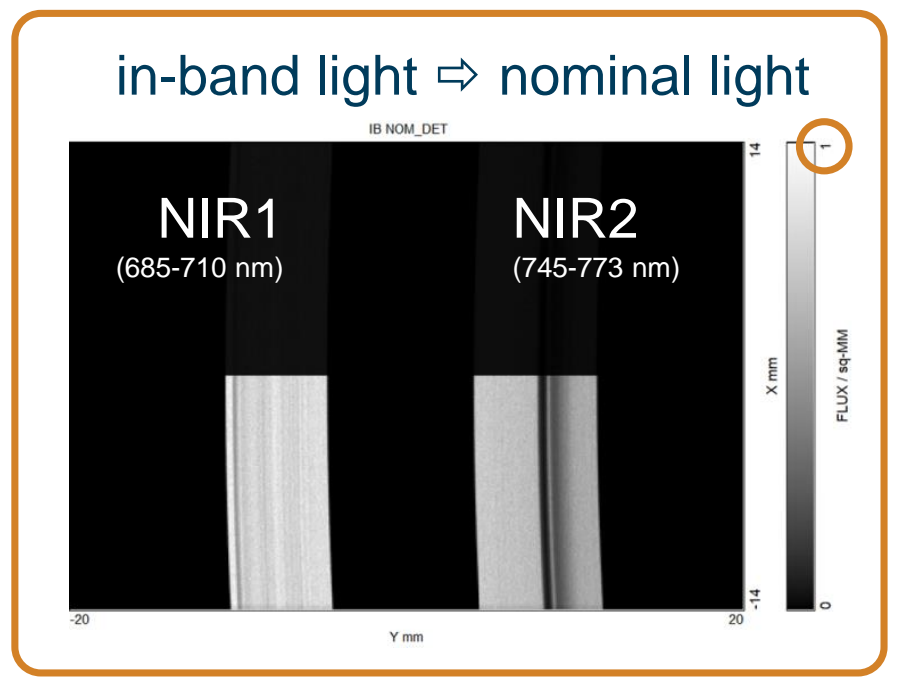

out-of-band light $\Rightarrow$ direct light

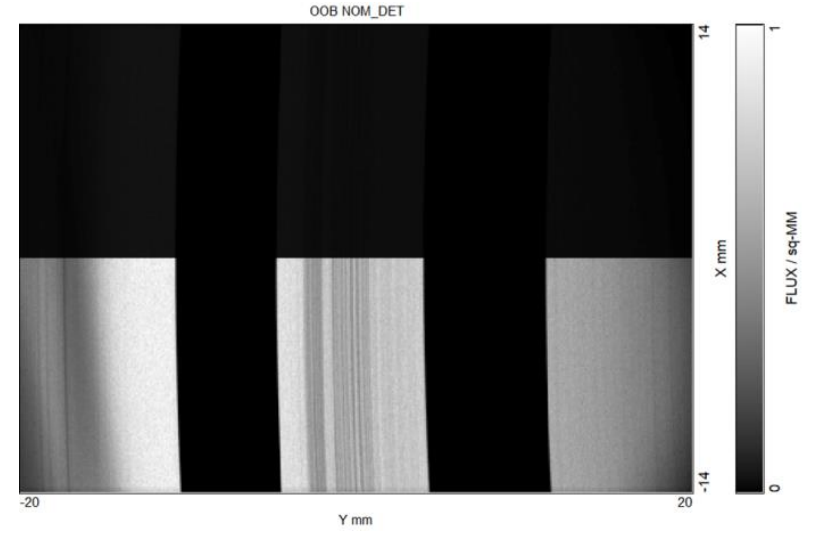

7 space for success 


\section{Ghost Analysis}

- Full input spectrum (520 nm - $930 \mathrm{~nm})$

- up to second order reflections (except for additional TIR)
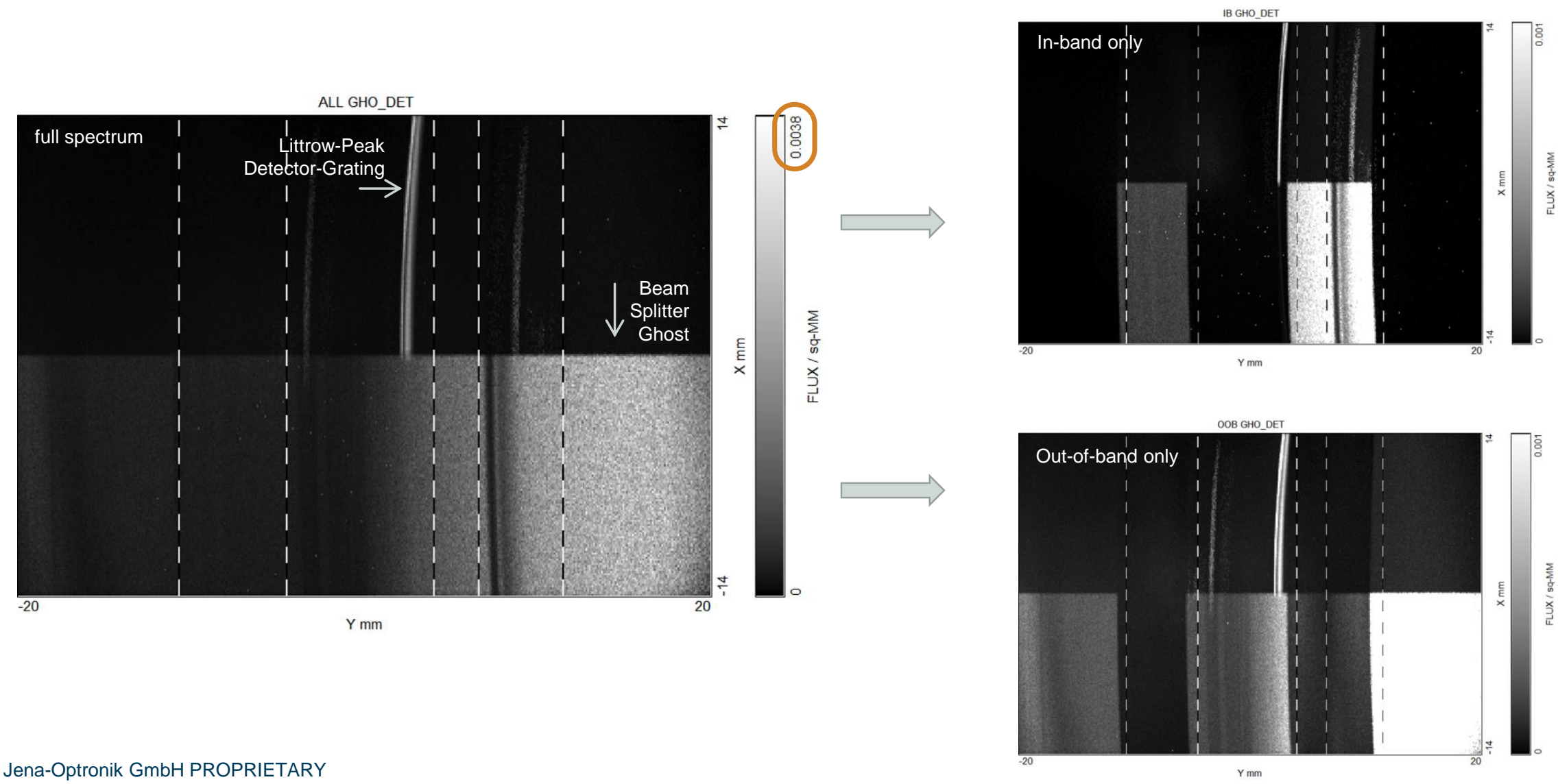


\section{Ghost Analysis}

- Selected wavelength bands
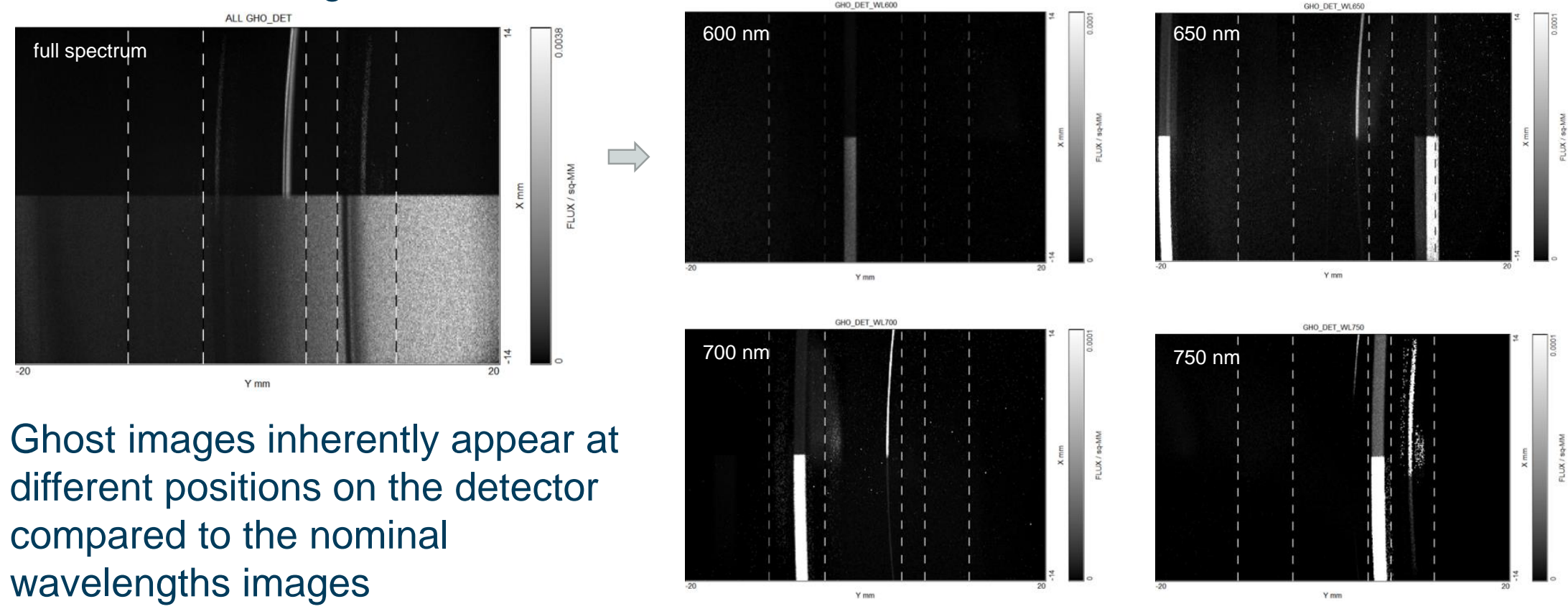

- Ghost images inherently appear at different positions on the detector compared to the nominal wavelengths images

$\Rightarrow$ shifts from out-of-band light into in-band detector regions!

- Some ghosts appear for specific wavelengths only, others are observable throughout the spectrum

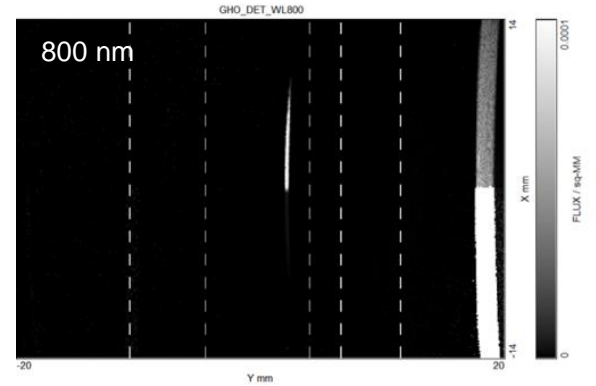

$850 \mathrm{~nm}$

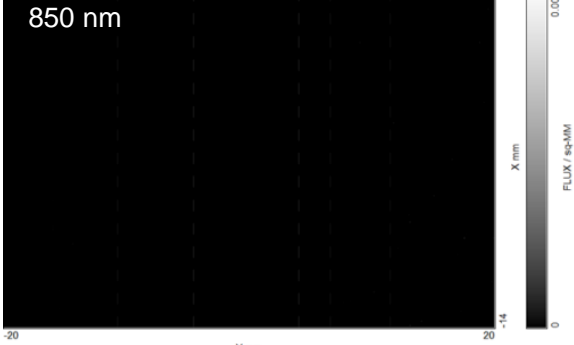




\section{Ghost Analysis}

- Path analysis

- separated by sequence (combination) of involved elements

- sortable by flux

- about 9000 different ghost paths identified; but most of them are negligible due to very low flux values

- some ghosts are inverted (flipped along spatial direction)

- some ghost are comparable sharp, others are widely spread

- important ghosts are evaluated for the 'dark side' of the detector

- clearly strongest path is generated by the beam splitter internal reflections

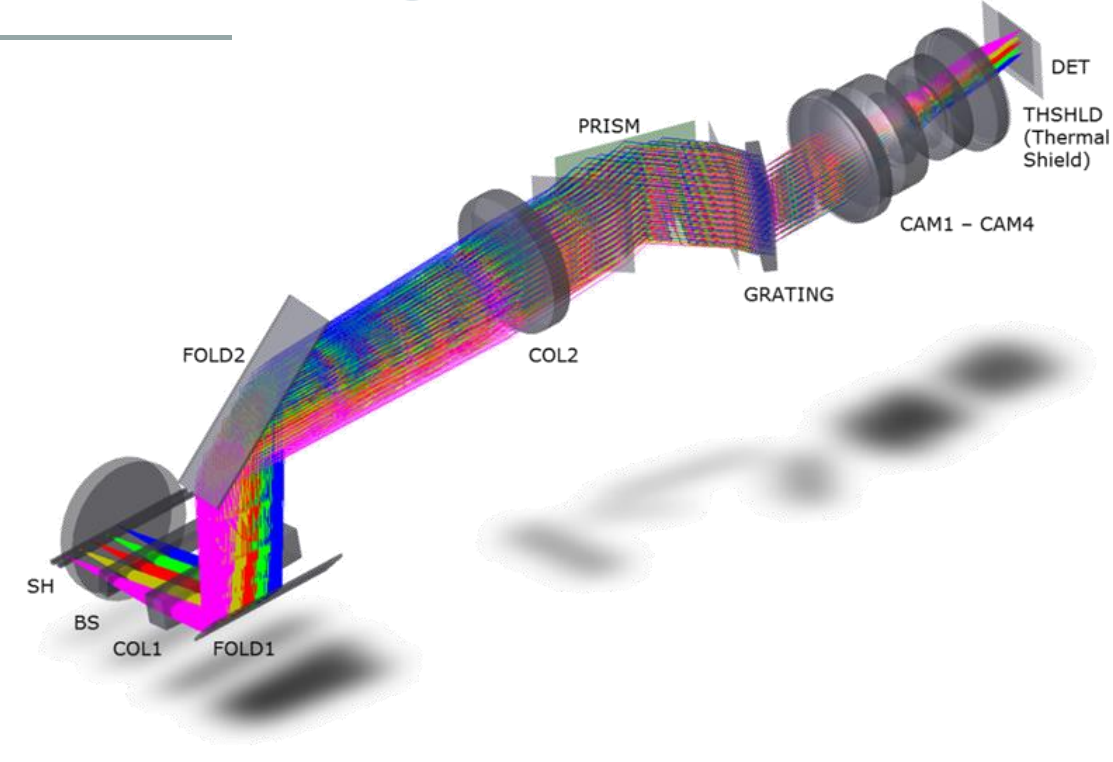

\begin{tabular}{|l|l|l|l|}
\hline$\#$ & SPLIT 1 & SPLIT 2 & SPLIT 3 \\
\hline 1 & BS (B) & BS (A) & \\
\hline 2 & DET & CAM4 (A) & \\
\hline 3 & CAM4 (A) & CAM3 face (A) & \\
\hline 4 & DET & CAM1 (B) & \\
\hline 5 & CAM1 (B) & PRISM (C) & GRATING \\
\hline 6 & CAM3 (B) & GRATING & \\
\hline 7 & DET & CAM3 (B) & \\
\hline & $\vdots$ & $\vdots$ & \\
\hline
\end{tabular}


- Example \#1: Beam splitter ghost
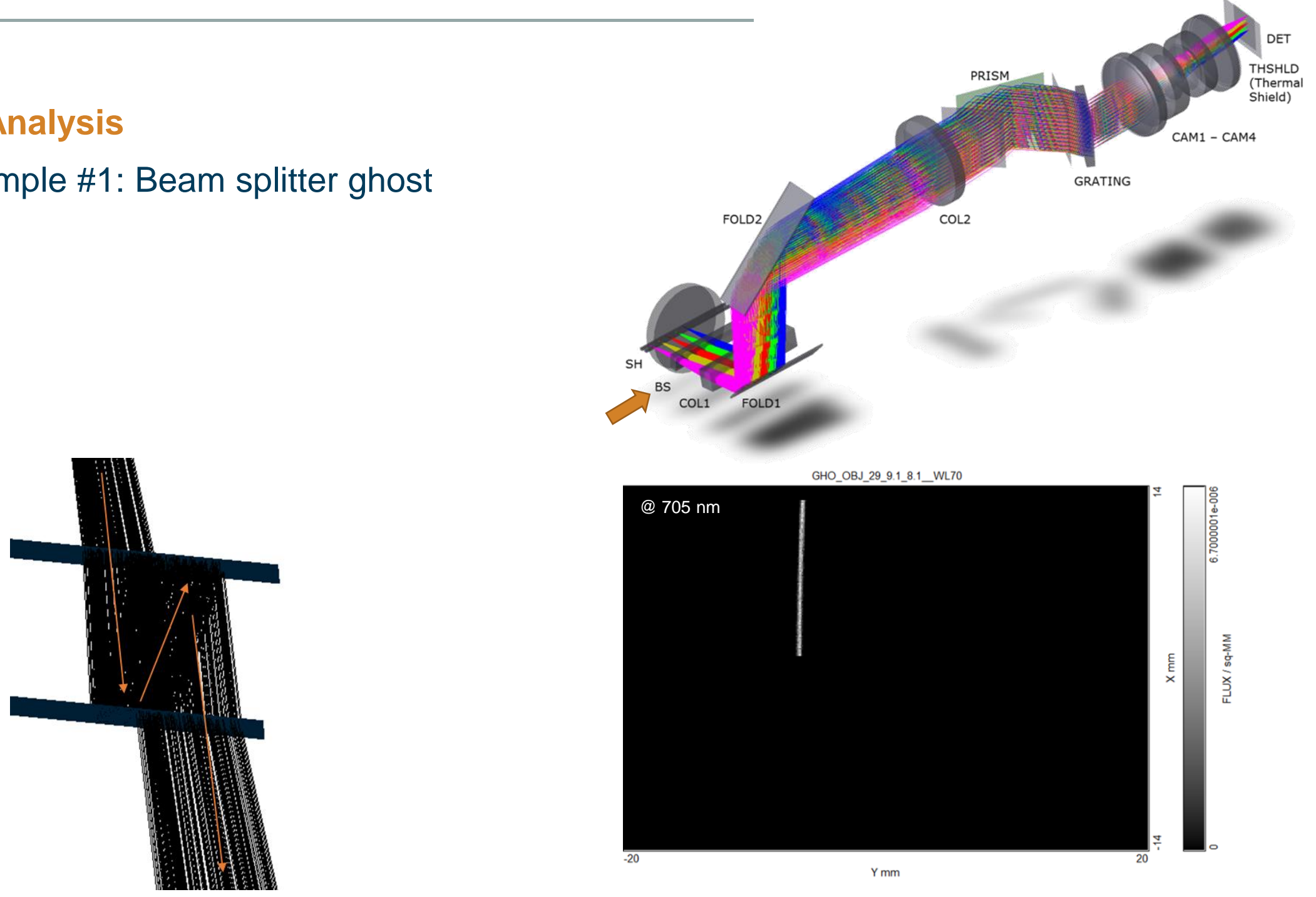

- Note: shown for dark side of detector and single wavelength only! 
- Example \#2: DET - CAM4 (A)
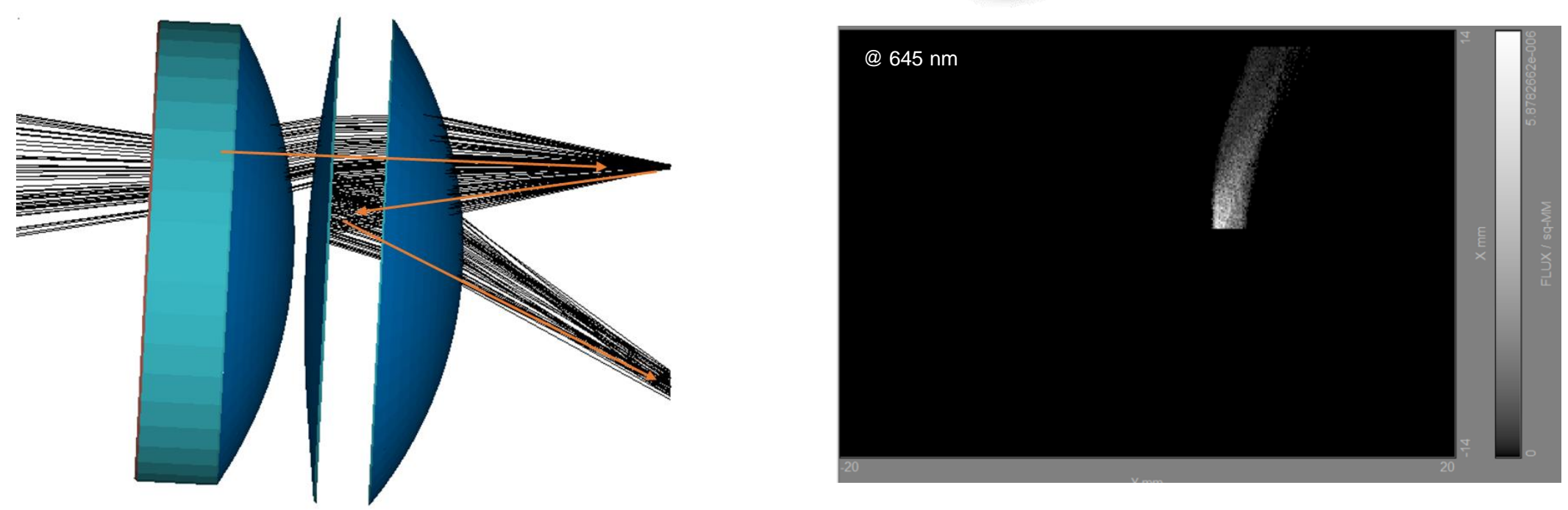

- Note: shown for dark side of detector and single wavelength only! 
Ghost Analysis

- Example \#5: CAM1 (B) - PRISM (C) - GRATING
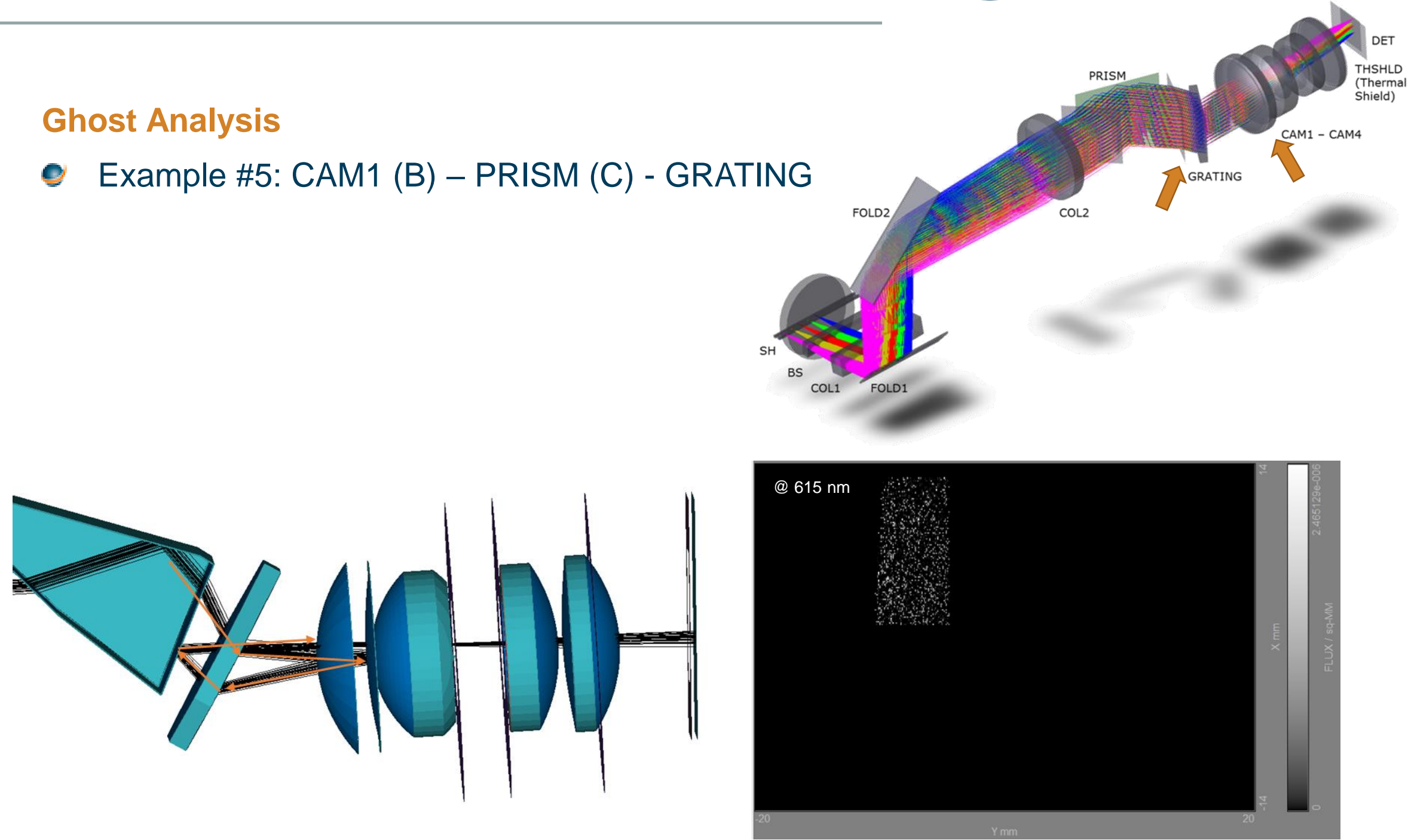

- Note: shown for dark side of detector and single wavelength only! 


\section{Scattering from Particle Contamination}

- Particle contamination scattering takes place at every optical surface

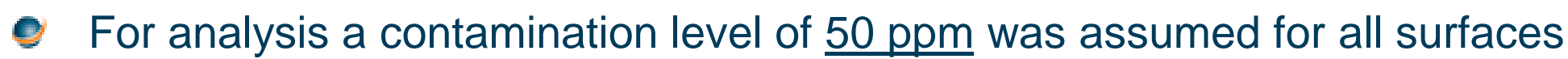

- BSDF model is sum of four 3-parametric Harvey models (based on Mie scattering)

- Total integrated scatter (TIS) is about $0.01 \%$

- Scattering is dominated by small-angle scattering (0 - 2 degrees wrt nominal beam) $\Rightarrow$ results in blurring of the nominal scene (about 1:1000 in contrast)

test scene with full input spectrum

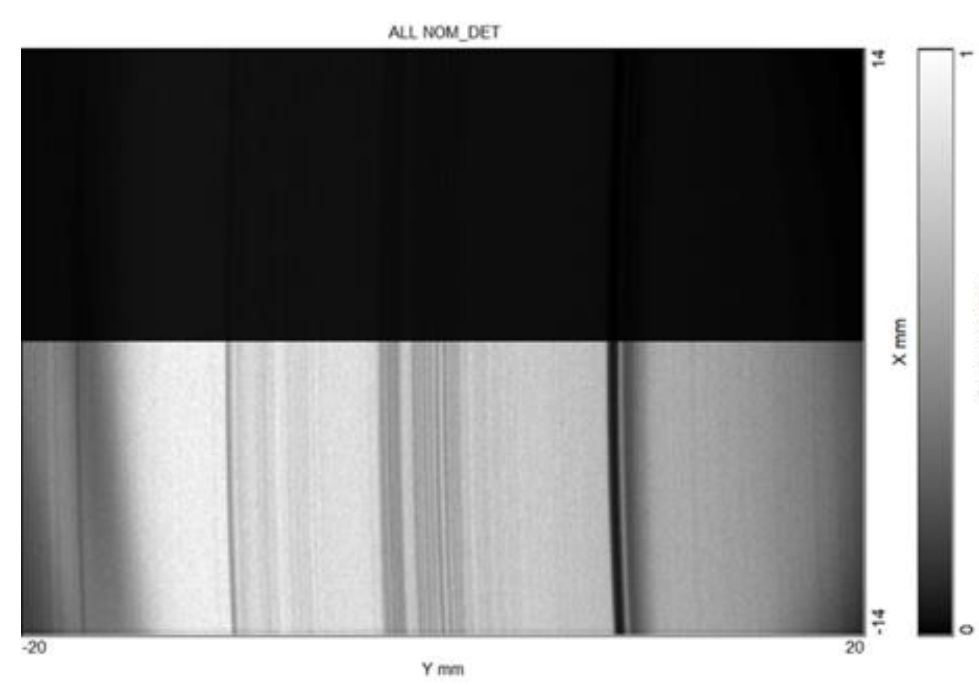

scattering from particle contamination

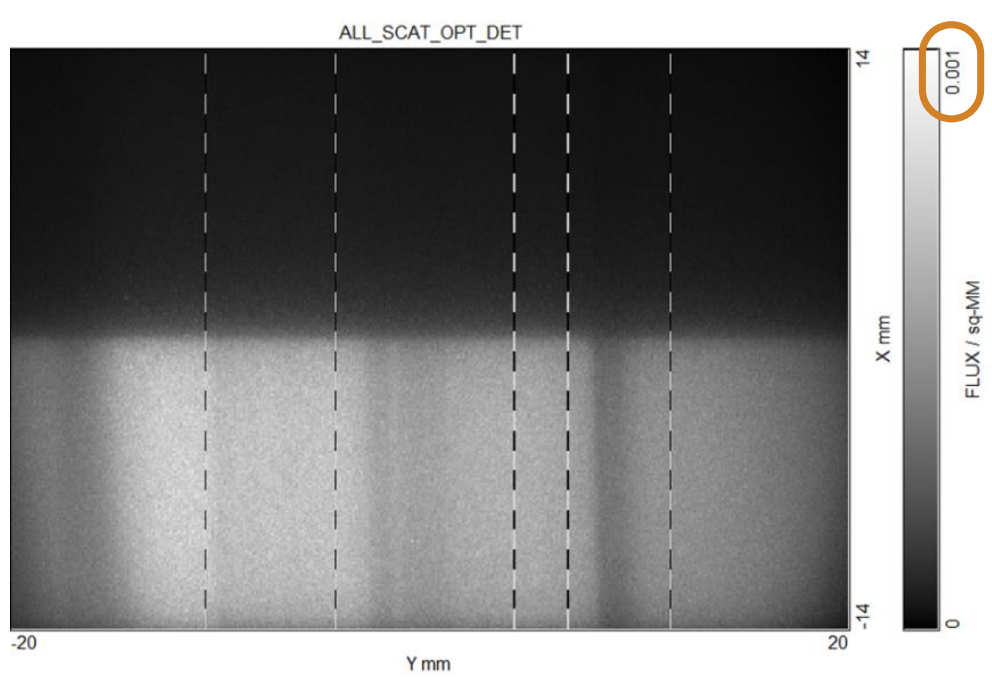




\section{Scattering from Surface Roughness}

- For analysis an roughness value of $1 \mathrm{~nm}$ RMS was assumed for most surfaces

- BSDF model is an adapted 3-parametric Harvey model (specified by customer) $\Rightarrow$ inside medium with higher impact (wavelength versus surface profile)

$\Rightarrow$ impact twice in reflection instead transmission (doubled paths differences)

- Scattering is dominated by small-angle scattering ( $0-0.5$ degrees) $\Rightarrow$ results in blurring of the nominal scene (about 1:500 in contrast)

test scene with full input spectrum

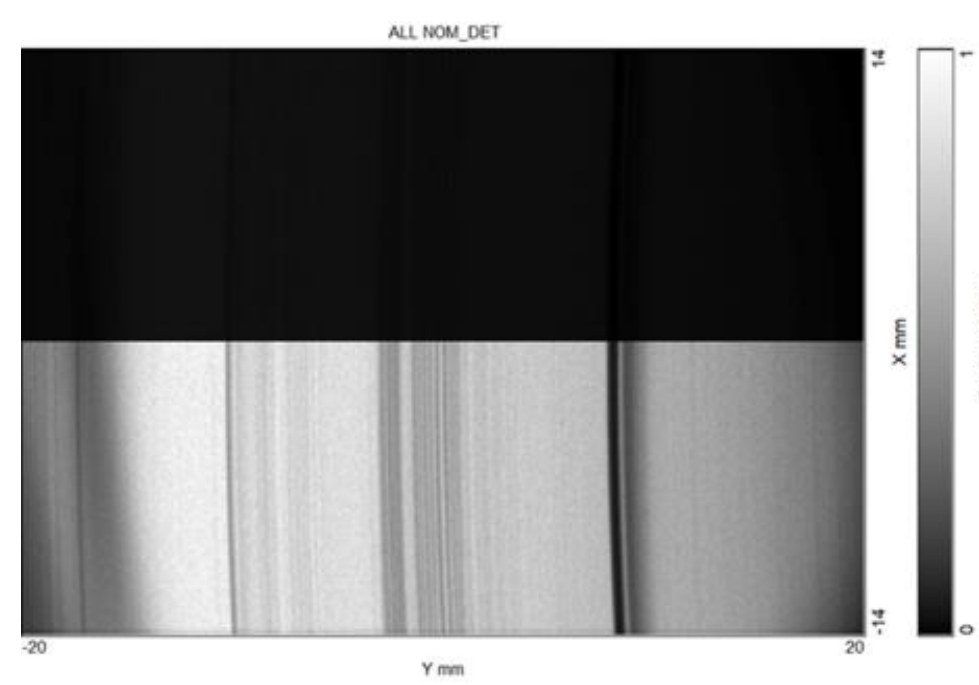

scattering from surface roughness

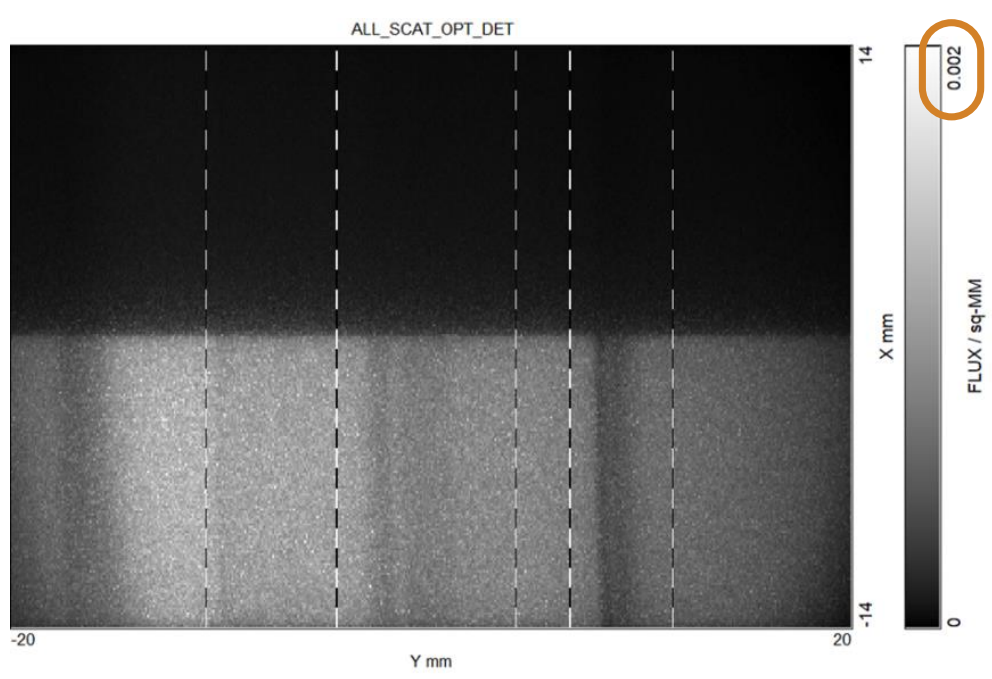




\section{Scattering from Mechanical Surfaces}

- Scatter models used in analysis based on different categories of surfaces:

(A) black coated surfaces $\rightarrow$ Acktar Magic Black ${ }^{\text {tm }}$ (TIS about 1.2\% for AOI of Odeg)

(B) uncoated parts $\rightarrow$ model based on assumptions for bare metal (TIS about 55\%)

(C) glue pads $\rightarrow$ model based on TIS measurements (TIS about 30\%)

(D) special parts with individual scattering behaviour: grating stop, thermal shield

- First order scattering only; detector reflection and all grating orders included

test scene with full input spectrum

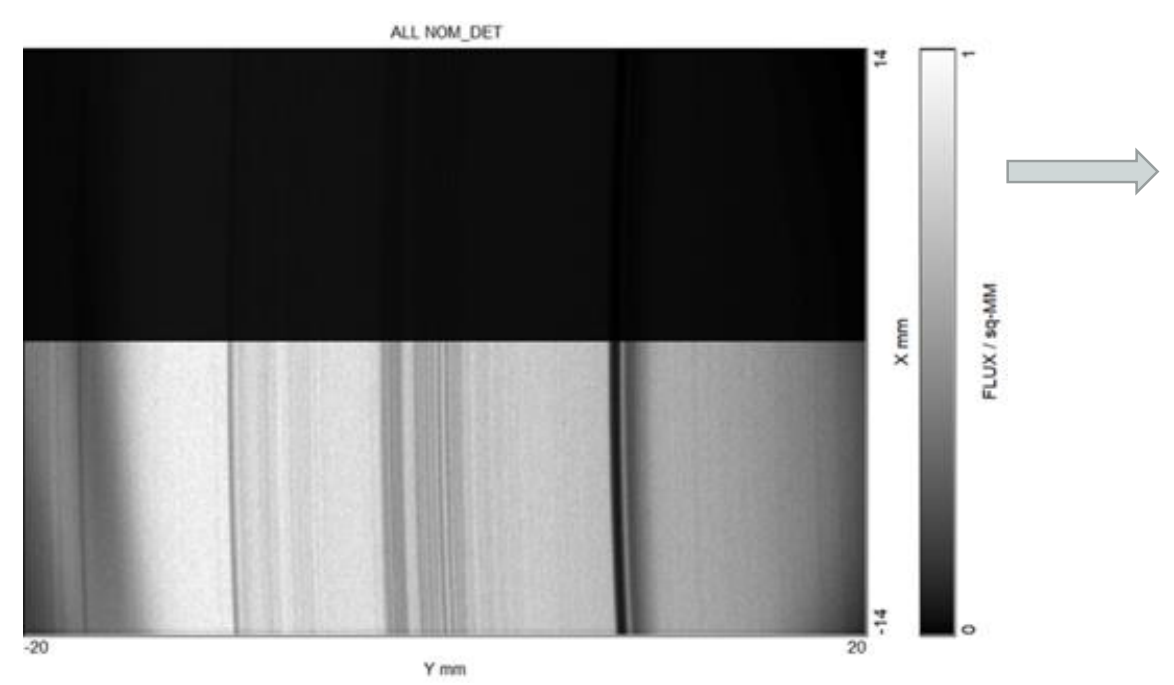

scattering from mechanical surfaces

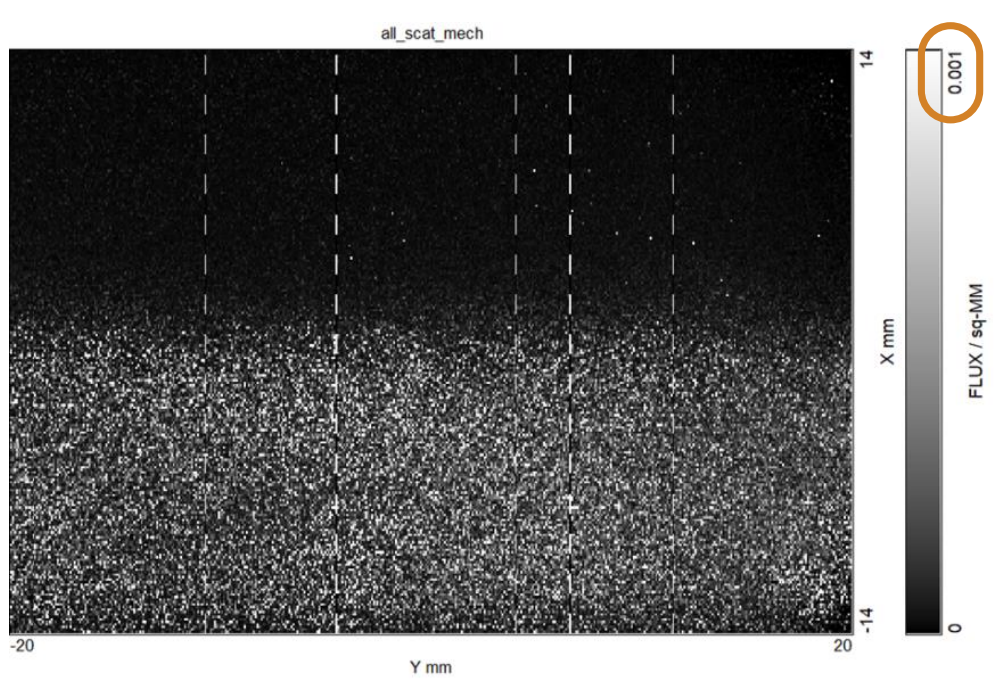




\section{Scattering from Mechanical Surfaces}

- Path analysis

- similar to ghost path analysis

- most critical parts are stop edges (forward scattering due to grazing incidence)

- Examples:
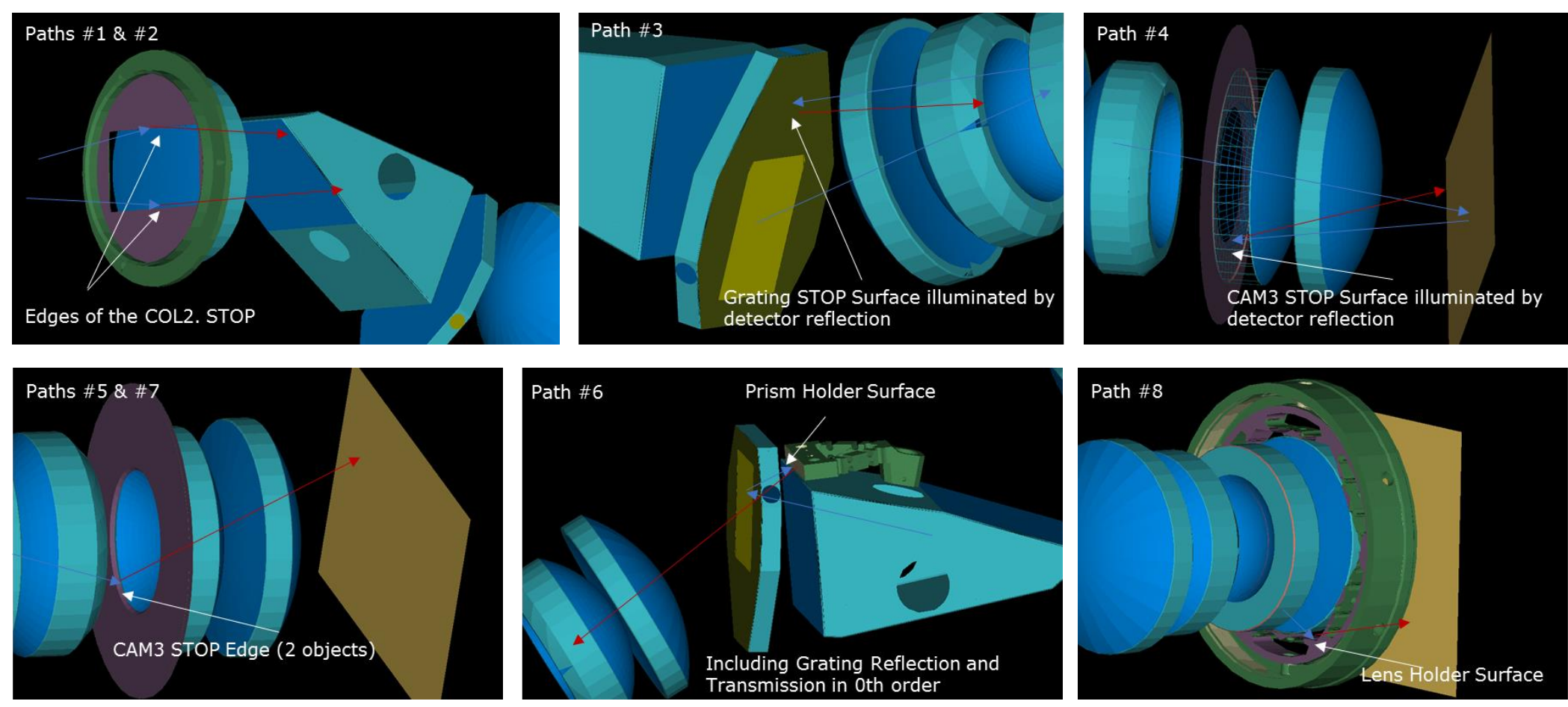


\section{Design improvements}

- Most critical contributors are given by customer and cannot be improved directly

- beam splitter design and spectral characteristics (up to $7 \%$ reflectivity at upper band limit of NIR2)

- reflectivity of detector (up to $8 \%$ at upper band limit of NIR2)

- reflectivity and scattering behaviour of thermal shield

- The main contributors can be categorized as in-field, in-band as well as out-of-band false light $\rightarrow$ full suppression is hard to achieve

- Major improvement due to reduction of outof-band light

$\Rightarrow$ fold mirrors made of absorbing NG5 and coated with adapted multi-layer stack (instead of simple metal coating)
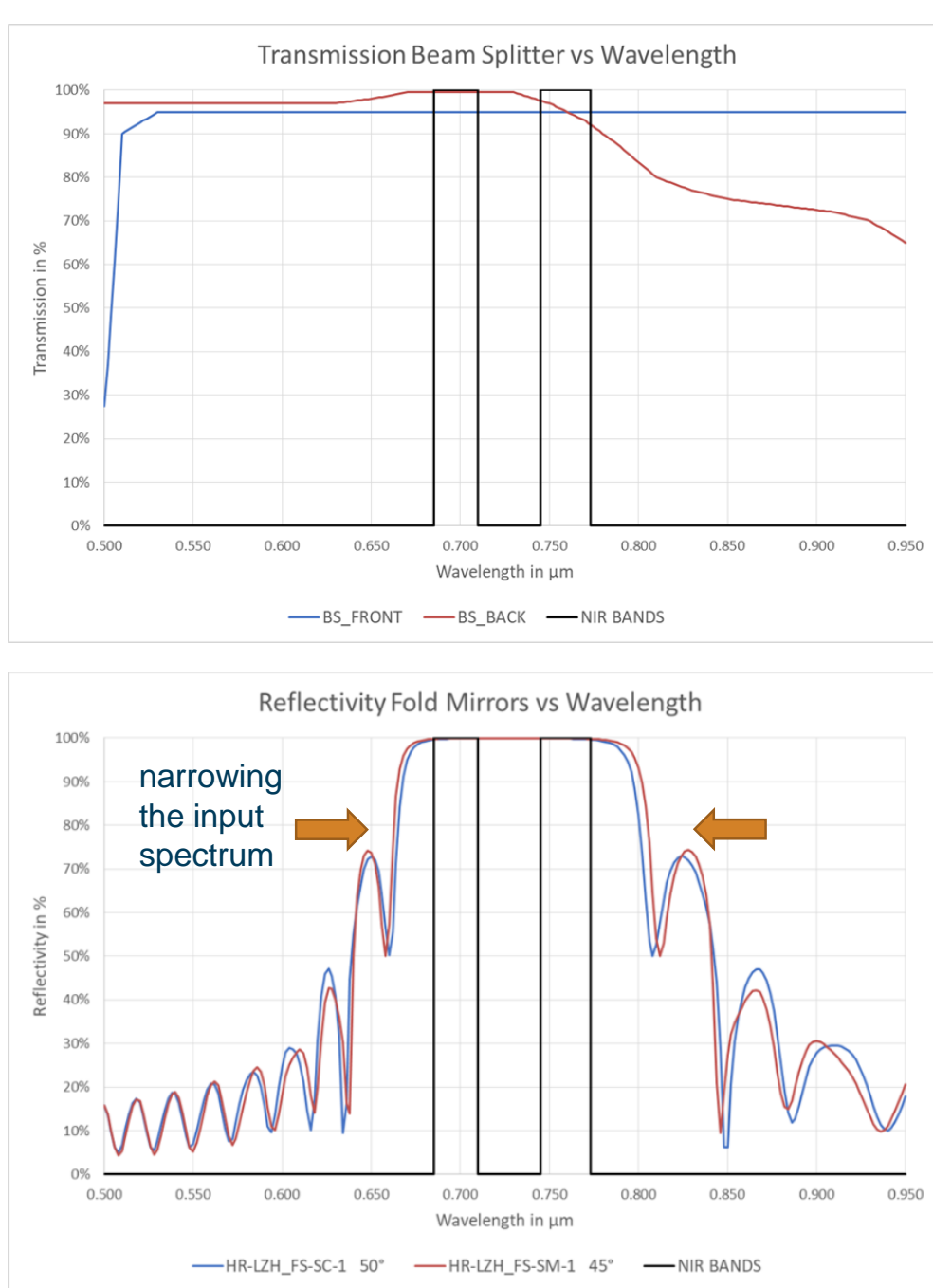


\section{jenaoptronık}

\section{Conclusion}

- The Sentinel-5 NIR spectrometer opto-mechanics are mainly driven by false light

- False light contributors were investigated

- Ghost light $\Rightarrow$ most dominant

- beam splitter with strongest impact

- First order scattering from optical components caused by

- surface roughness (55\% of total scattering)

- particle contamination (30\% of total scattering)

- First order scattering from mechanical parts (15\% of total scattering)

- Present design in terms of cleanliness (particle contamination), surface polishing (roughness) and black coating (Acktar Magic Black) is almost state of the art

- Major improvement via implementation of fold mirrors with adapted multi-layer coatings and absorbing substrates (reducing out-of-band false light)

- Suggestion for further improvements

- design adaption of beam splitter coatings

- high-performance broad-band anti-reflection coating for detector 


\section{Acknowledgements}

- Main parts of this analysis were performed by Hembach Photonik $\mathrm{GmbH}$, namely Bernd Harand, Bernhard Michel, Robert Fischer, and Davit Sivil

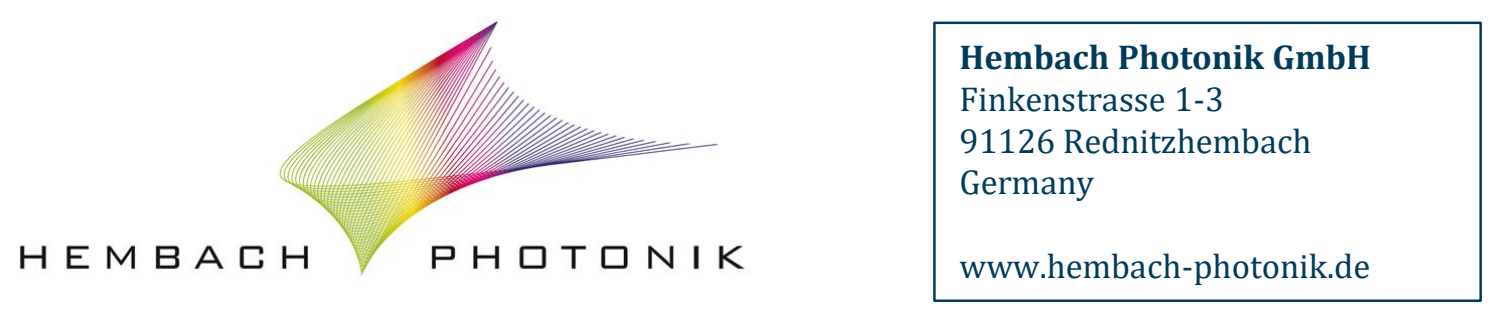

- Many thanks for their contribution and fruitful discussions: Matthias Merschdorf, Systems Engineering, Jena-Optronik GmbH Lukasz Platos, Optics Engineering, Jena-Optronik GmbH

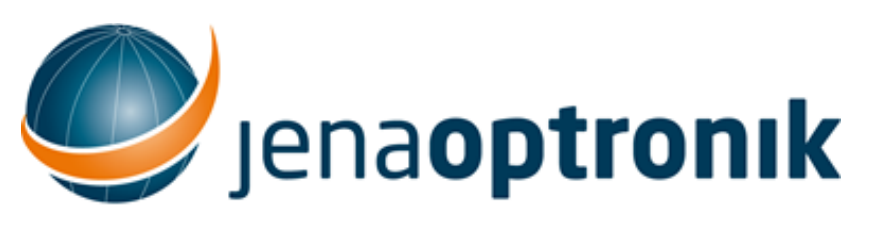

